\title{
Improved Exponential Estimator in Stratified Random Sampling
}

\author{
Rajesh Singh \\ Department of Statistics \\ B.H.U., Varanasi (U.P.) \\ India \\ rsinghstat@yahoo.com \\ Mukesh Kumar \\ Department of Statistics \\ B.H.U., Varanasi (U.P.) \\ India \\ Manoj K. Chaudhary \\ Department of Statistics \\ B.H.U., Varanasi (U.P.) \\ India

\section{Cem Kadilar \\ Department of Statistics \\ Hacettepe University \\ Beytepe 06800, Ankara \\ Turkey \\ kadilar@hacettepe.edu.tr}

\begin{abstract}
In this article we have considered the problem of estimating the population mean $(\bar{Y})$ in the stratified random sampling using the information of an auxiliary variable $\mathrm{x}$ which is correlated with $y$ and suggested improved exponential ratio estimators in the stratified random sampling. The mean square error (MSE) equations for the proposed estimators have been derived and it is shown that the proposed estimators under optimum condition performs better than estimators suggested by Singh et al. (2008). Theoretical and empirical findings are encouraging and support the soundness of the proposed estimators for mean estimation.
\end{abstract}

Keywords: Auxiliary variable, exponential ratio-type estimates, mean square error, stratified random sampling.

2000 AMS Classification: 62D05

\section{Introduction}

In survey sampling, it is well established that the use of auxiliary information results in substantial gain in efficiency over the estimators which do not use such information. However, in planning surveys, the stratified sampling has often proved needful in improving the precision of estimates over simple random sampling. In some cases, in addition to mean of auxiliary variable, various parameters related to auxiliary variable such as coefficient of variation, kurtosis, correlation coefficient, etc. may also be known. For these cases, many authors, 
such as Upadhayaya and Singh (1999), Sisodia and Dwivedi (1981), Singh and Tailor (2003), Singh et al. (2007), developed various estimators to improve the ratio estimators in the simple random sampling. Kadilar and Cingi (2003) adapted the estimators in Upadhyaya and Singh (1999) to the stratified random sampling.

In this study, under stratified random sampling without replacement scheme, we suggest two improved exponential estimators which are more efficient than estimators proposed by Singh et al. (2008). There are some recent studies proposing the family of estimators without using the exponential function in literature, such as Koyuncu and Kadilar (2009(a,b)), Khoshnevisan et al. (2007), Singh and Vishwakarma $(2006,2008)$ etc. However, in this article, the proposed family of estimators depends on the exponential function.

We assume that the population comprises $\mathrm{N}$ units, which can be uniquely partitioned into $L$ strata of size $N_{1}, N_{2}, \ldots, N_{L}$ such that $\sum_{h=1}^{L} N_{h}=N$. The strata weights $W_{h}=N_{h} / N(h=1,2, \ldots, L)$ are assumed known. Let $\left(y_{h i}, x_{h i}\right)\left(i=1,2, \ldots, N_{h}\right)$ denote the values of variates $(\mathrm{y}, \mathrm{x})$ respectively for the $\mathrm{i}$-th unit in the $\mathrm{h}$-th stratum and $\bar{Y}_{\mathrm{h}}$ and $\overline{\mathrm{X}}_{\mathrm{h}}$ denote stratum means.

When the population mean of the auxiliary variable, $\bar{X}$, is known, Hansen et al. (1946) suggested a combined ratio estimator for estimating the population mean of the study variable $(\overline{\mathrm{Y}})$ :

$$
\mathrm{t}_{1}=\frac{\overline{\mathrm{y}}_{\mathrm{st}}}{\overline{\mathrm{x}}_{\mathrm{st}}} \overline{\mathrm{X}}
$$

where $\overline{\mathrm{y}}_{\mathrm{st}}=\sum_{\mathrm{h}=1}^{\mathrm{L}} \mathrm{w}_{\mathrm{h}} \overline{\mathrm{y}}_{\mathrm{h}}, \quad \overline{\mathrm{x}}_{\mathrm{st}}=\sum_{\mathrm{h}=1}^{\mathrm{L}} \mathrm{w}_{\mathrm{h}} \overline{\mathrm{x}}_{\mathrm{h}}$, and $\overline{\mathrm{X}}=\sum_{\mathrm{h}=1}^{\mathrm{L}} \mathrm{w}_{\mathrm{h}} \overline{\mathrm{X}}_{\mathrm{h}}$.

Here $\bar{y}_{h}=\frac{1}{n_{h}} \sum_{i=1}^{n_{h}} y_{h i}, \quad$ and $\quad \bar{x}_{h}=\frac{1}{n_{h}} \sum_{i=1}^{n_{h}} x_{h i}$.

The MSE of $t_{1}$ to a first degree of approximation is given by

$$
\operatorname{MSE}\left(\mathrm{t}_{1}\right) \cong \sum_{\mathrm{h}=1}^{\mathrm{L}} \mathrm{w}_{\mathrm{h}}^{2} \gamma_{\mathrm{h}}\left[\mathrm{S}_{\mathrm{yh}}^{2}+\mathrm{R}^{2} \mathrm{~S}_{\mathrm{xh}}^{2}-2 \mathrm{RS} \mathrm{yxh}_{\mathrm{y}}\right]
$$

where $\gamma_{\mathrm{h}}=\left(\frac{1}{\mathrm{n}_{\mathrm{h}}}-\frac{1}{\mathrm{~N}_{\mathrm{h}}}\right), \quad \mathrm{R}=\frac{\overline{\mathrm{Y}}}{\overline{\mathrm{X}}}=\frac{\overline{\mathrm{Y}}_{\mathrm{st}}}{\overline{\mathrm{X}}_{\mathrm{st}}}$ is the population ratio, $\mathrm{S}_{\mathrm{yh}}^{2}$ is the population variance of a study variable, $\mathrm{S}_{\mathrm{xh}}^{2}$ is the population variance of the auxiliary variable and $S_{y x h}$ is the population covariance between study and auxiliary variables in the stratum $\mathrm{h}$. 


\section{Kadilar and Cingi estimator}

Kadilar and Cingi (2003) introduced an estimator for population mean using known value of some population parameters in stratified random sampling given by

$$
\mathrm{t}_{2}=\frac{\overline{\mathrm{y}}_{\mathrm{st}}}{\overline{\mathrm{x}}_{\mathrm{st}, \mathrm{a}, \mathrm{b}}} \overline{\mathrm{X}}_{\mathrm{st}, \mathrm{a}, \mathrm{b}}
$$

where $\overline{\mathrm{x}}_{\text {st }, \mathrm{a}, \mathrm{b}}=\sum_{\mathrm{h}=1}^{\mathrm{L}} \mathrm{w}_{\mathrm{h}}\left(\mathrm{a}_{\mathrm{h}} \overline{\mathrm{x}}_{\mathrm{h}}+\mathrm{b}_{\mathrm{h}}\right), \quad \overline{\mathrm{X}}_{\mathrm{st}, \mathrm{a}, \mathrm{b}}=\sum_{\mathrm{h}=1}^{\mathrm{L}} \mathrm{w}_{\mathrm{h}}\left(\mathrm{a}_{\mathrm{h}} \overline{\mathrm{x}}_{\mathrm{h}}+\mathrm{b}_{\mathrm{h}}\right)$ and $\mathrm{a}_{\mathrm{h}}, \mathrm{b}_{\mathrm{h}}$ are the functions of the known parameters of the auxiliary variable such as coefficient of variation $C_{x h}$, coefficient of kurtosis $\beta_{2 h}$ (x)etc., or some constants in the $h^{\text {th }}$ stratum .

The MSE of the estimator $t_{2}$ is given by

$$
\operatorname{MSE}\left(\mathrm{t}_{2}\right)=\sum_{\mathrm{h}=1}^{\mathrm{L}} \mathrm{W}_{\mathrm{h}}^{2} \gamma_{\mathrm{h}}\left[\mathrm{S}_{\mathrm{yh}}^{2}+\mathrm{R}_{\mathrm{a}, \mathrm{b}}^{2} \mathrm{a}_{\mathrm{h}}^{2} \mathrm{~S}_{\mathrm{xh}}^{2}-2 \mathrm{R}_{\mathrm{a}, \mathrm{b}} \mathrm{a}_{\mathrm{h}} \mathrm{S}_{\mathrm{yxh}}\right] \text {, }
$$

where $R_{a, b}=\frac{\bar{Y}_{s t}}{\bar{X}_{s t, a, b}}$.

Bahl and Tuteja (1991) suggested an exponential ratio type estimator for population mean in simple random sampling as

$$
\mathrm{t}_{3}=\overline{\mathrm{y}} \exp \left[\frac{\overline{\mathrm{X}}-\overline{\mathrm{x}}}{\overline{\mathrm{X}}+\overline{\mathrm{x}}}\right] \text {. }
$$

Motivated by Bahl and Tuteja (1991), Singh et al. (2008) adapted this estimator to the stratified random sampling as

$$
\mathrm{t}_{4 \mathrm{i}}=\overline{\mathrm{y}}_{\mathrm{st}} \exp \left[\frac{\overline{\mathrm{X}}_{4 \mathrm{i}}-\overline{\mathrm{x}}_{4 \mathrm{i}}}{\overline{\mathrm{X}}_{4 \mathrm{i}}+\overline{\mathrm{x}}_{4 \mathrm{i}}}\right], \quad \mathrm{i}=0,1,2,3,4 .
$$

where

$$
\begin{aligned}
\overline{\mathrm{x}}_{40}=\sum_{\mathrm{h}=1}^{\mathrm{L}} \mathrm{w}_{\mathrm{h}} \overline{\mathrm{x}}_{\mathrm{h}}, & \overline{\mathrm{X}}_{40}=\sum_{\mathrm{h}=1}^{\mathrm{L}} \mathrm{w}_{\mathrm{h}} \overline{\mathrm{X}}_{\mathrm{h}}, \\
\overline{\mathrm{x}}_{41}=\sum_{\mathrm{h}=1}^{\mathrm{L}} \mathrm{w}_{\mathrm{h}}\left(\overline{\mathrm{x}}_{\mathrm{h}}+\mathrm{C}_{\mathrm{xh}}\right), & \overline{\mathrm{X}}_{41}=\sum_{\mathrm{h}=1}^{\mathrm{L}} \mathrm{w}_{\mathrm{h}}\left(\overline{\mathrm{X}}_{\mathrm{h}}+\mathrm{C}_{\mathrm{xh}}\right), \\
\overline{\mathrm{x}}_{42}=\sum_{\mathrm{h}=1}^{\mathrm{L}} \mathrm{w}_{\mathrm{h}}\left[\overline{\mathrm{x}}_{\mathrm{h}}+\beta_{2 \mathrm{~h}}(\mathrm{x})\right], & \overline{\mathrm{X}}_{42}=\sum_{\mathrm{h}=1}^{\mathrm{L}} \mathrm{w}_{\mathrm{h}}\left[\overline{\mathrm{X}}_{\mathrm{h}}+\beta_{2 \mathrm{~h}}(\mathrm{x})\right], \\
\overline{\mathrm{x}}_{43}=\sum_{\mathrm{h}=1}^{\mathrm{L}} \mathrm{w}_{\mathrm{h}}\left[\overline{\mathrm{x}}_{\mathrm{h}} \beta_{2 \mathrm{~h}}(\mathrm{x})+\mathrm{C}_{\mathrm{xh}}\right], & \overline{\mathrm{X}}_{43}=\sum_{\mathrm{h}=1}^{\mathrm{L}} \mathrm{w}_{\mathrm{h}}\left[\overline{\mathrm{X}}_{\mathrm{h}} \beta_{2 \mathrm{~h}}(\mathrm{x})+\mathrm{C}_{\mathrm{xh}}\right], \\
\overline{\mathrm{x}}_{44}=\sum_{\mathrm{h}=1}^{\mathrm{L}} \mathrm{w}_{\mathrm{h}}\left[\overline{\mathrm{x}}_{\mathrm{h}} \mathrm{C}_{\mathrm{xh}}+\beta_{2 \mathrm{~h}}(\mathrm{x})\right], & \overline{\mathrm{X}}_{44}=\sum_{\mathrm{h}=1}^{\mathrm{L}} \mathrm{w}_{\mathrm{h}}\left[\overline{\mathrm{X}}_{\mathrm{h}} \mathrm{C}_{\mathrm{xh}}+\beta_{2 \mathrm{~h}}(\mathrm{x})\right] .
\end{aligned}
$$


The bias and MSE of the estimators $t_{4 i}(i=0,1,2,3,4)$ can be obtained respectively by following expressions:

$$
\begin{aligned}
& \operatorname{Bias}\left(\mathrm{t}_{4 \mathrm{i}}\right)=\frac{1}{\bar{X}_{4 i}} \sum_{h=1}^{\mathrm{L}} \mathrm{w}_{\mathrm{h}}^{2} \gamma_{\mathrm{h}}\left(\frac{3}{8} \mathrm{R}_{4 \mathrm{i}} \mathrm{a}_{\mathrm{hi}}^{2} \mathrm{~S}_{\mathrm{xh}}^{2}-\frac{1}{2} \mathrm{a}_{\mathrm{hi}} \mathrm{S}_{\mathrm{yxh}}\right),(\mathrm{i}=01,2,3,4) \\
& \operatorname{MSE}\left(\mathrm{t}_{4 \mathrm{i}}\right)=\sum_{\mathrm{h}=1}^{\mathrm{L}} \mathrm{w}_{\mathrm{h}}^{2} \gamma_{\mathrm{h}}\left(\mathrm{S}_{\mathrm{yh}}^{2}-\mathrm{R}_{4 \mathrm{i}} \mathrm{a}_{\mathrm{hi}} \mathrm{S}_{\mathrm{yxh}}+\frac{\mathrm{R}_{4 \mathrm{i}}^{2}}{4} \mathrm{a}_{\mathrm{hi}}^{2} \mathrm{~S}_{\mathrm{xh}}^{2}\right),(\mathrm{i}=01,2,3,4)
\end{aligned}
$$

where,

$$
\begin{aligned}
& \mathrm{R}_{40}=\frac{\overline{\mathrm{Y}}_{\mathrm{st}}}{\sum_{\mathrm{h}=1}^{\mathrm{L}} \mathrm{w}_{\mathrm{h}} \overline{\mathrm{X}}_{\mathrm{h}}}
\end{aligned}
$$

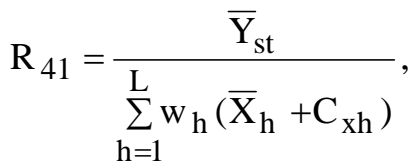

$$
\begin{aligned}
& \mathrm{R}_{42}=\frac{\overline{\mathrm{Y}}_{\mathrm{st}}}{\sum_{\mathrm{h}=1}^{\mathrm{L}} \mathrm{w}_{\mathrm{h}}\left(\overline{\mathrm{X}}_{\mathrm{h}}+\beta_{2 \mathrm{~h}}(\mathrm{x})\right)},
\end{aligned}
$$

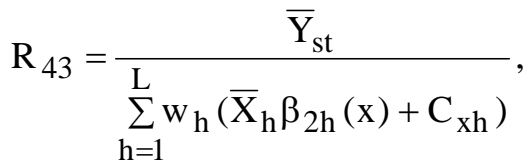

$$
\begin{aligned}
& \mathrm{R}_{44}=\frac{\overline{\mathrm{Y}}_{\mathrm{st}}}{\sum_{\mathrm{h}=1}^{\mathrm{L} \mathrm{w}_{\mathrm{h}}}\left(\overline{\mathrm{X}}_{\mathrm{h}} \mathrm{C}_{\mathrm{xh}}+\beta_{2 \mathrm{~h}}(\mathrm{x})\right)} \text {, } \\
& \overline{\mathrm{Y}}_{\mathrm{st}}=\sum_{\mathrm{h}=1}^{\mathrm{L}} \mathrm{w}_{\mathrm{h}} \overline{\mathrm{Y}}_{\mathrm{h}}=\overline{\mathrm{Y}} \text {. }
\end{aligned}
$$

We would like to remark that for various values of parameters in (2.4), we get five

\begin{tabular}{|c|c|c|}
\hline $\begin{array}{c}\text { The values } \\
\text { of } a_{h}, b_{h}\end{array}$ & Estimator & MSE of the Estimator \\
\hline$a_{h}=1, b_{h}=0$ & $\mathrm{t}_{40}=\overline{\mathrm{y}}_{\mathrm{st}} \exp \left[\frac{\overline{\mathrm{X}}-\overline{\mathrm{x}}_{\mathrm{st}}}{\overline{\mathrm{X}}+\overline{\mathrm{x}}_{\mathrm{st}}}\right]$ & $\sum_{h=1}^{L} w_{h}^{2} \gamma_{h}\left(S_{y h}^{2}-R_{40} S_{y x h}+\frac{R_{40}^{2}}{4} S_{x h}^{2}\right.$ \\
\hline $\begin{array}{l}a_{h}=1 \\
b_{h}=C_{x h}\end{array}$ & $\mathrm{t}_{41}=\overline{\mathrm{y}}_{\mathrm{st}} \exp \left[\frac{\overline{\mathrm{X}}_{41}-\overline{\mathrm{x}}_{41}}{\overline{\mathrm{X}}_{41}+\overline{\mathrm{x}}_{41}}\right]$ & $\sum_{h=1}^{L} w_{h}^{2} \gamma_{h}\left(S_{y h}^{2}-R_{41} S_{y x h}+\frac{R_{41}^{2}}{4} S_{x h}^{2}\right)$ \\
\hline $\begin{array}{l}a_{h}=1 \\
b_{h}=\beta_{2 h}(x)\end{array}$ & $\mathrm{t}_{42}=\overline{\mathrm{y}}_{\mathrm{st}} \exp \left[\frac{\overline{\mathrm{X}}_{42}-\overline{\mathrm{x}}_{42}}{\overline{\mathrm{X}}_{42}+\overline{\mathrm{x}}_{42}}\right.$ & $\sum_{h=1}^{L} w_{h}^{2} \gamma_{h}\left(S_{y h}^{2}-R_{42} S_{y x h}+\frac{R_{42}^{2}}{4} S_{x h}^{2}\right)$ \\
\hline $\begin{array}{l}a_{h}=\beta_{2 h}(x), \\
b_{h}=C_{x h}\end{array}$ & $\mathrm{t}_{43}=\overline{\mathrm{y}}_{\mathrm{st}} \exp \left[\frac{\overline{\mathrm{X}}_{43}-\overline{\mathrm{x}}_{43}}{\overline{\mathrm{X}}_{43}+\overline{\mathrm{x}}_{43}}\right]$ & $\sum_{h=1}^{L} w_{h}^{2} \gamma_{h}\left(S_{y h}^{2}-R_{43} \beta_{2 h}(x) S_{y x h}+\frac{R_{43}^{2}}{4} \beta_{2 h}^{2}(x) S_{x h}^{2}\right.$ \\
\hline $\begin{array}{l}a_{h}=C_{x h}, \\
b_{h}=\beta_{2 h}(x)\end{array}$ & $\mathrm{t}_{44}=\overline{\mathrm{y}}_{\mathrm{st}} \exp \left[\frac{\overline{\mathrm{X}}_{44}-\overline{\mathrm{x}}_{44}}{\overline{\mathrm{X}}_{44}+\overline{\mathrm{x}}_{44}}\right.$ & $\sum_{\mathrm{h}=1}^{\mathrm{L}} \mathrm{w}_{\mathrm{h}}^{2} \gamma_{\mathrm{h}}\left(\mathrm{S}_{\mathrm{yh}}^{2}-\mathrm{R}_{44} \mathrm{C}_{\mathrm{xh}} \mathrm{S}_{\mathrm{yxh}}+\frac{\mathrm{R}_{44}^{2}}{4} \mathrm{C}_{\mathrm{xh}}^{2} \mathrm{~S}_{\mathrm{xh}}^{2}\right)$ \\
\hline
\end{tabular}
estimators (for I = 0,1,2,3,4) as shown in Table 1. The MSE expressions for these estimators are also given in the Table 1.

Table 1: Some members of family of estimators $t_{4 i}$ 
Remark 1 - Here we would like to mention that the choice of the estimator depends on the availability and values of the various parameter(s) used (for choice of the parameters $a_{h}$ and $b_{h}$ refer to Singh et al. (2008) and Singh and Kumar(2009)).

Singh et al. (2008) showed that all of these modified estimators $\left(t_{4 i}\right)$ have a smaller MSE than the MSE of the stratified version of exponential ratio type estimator $\mathrm{t}_{3 \mathrm{st}}$ under certain conditions:

$$
\mathrm{t}_{3 \mathrm{st}}=\overline{\mathrm{y}}_{\mathrm{st}} \exp \left[\frac{\overline{\mathrm{X}}-\overline{\mathrm{x}}_{\mathrm{st}}}{\overline{\mathrm{X}}+\overline{\mathrm{x}}_{\mathrm{st}}}\right]
$$

Note that $t_{3 s t}$ is the same estimator with $t_{40}$.

Singh et al. (2008) reported the minimum value of mean square error of the estimator $t_{4 i}$ as $\operatorname{MSE}\left(t_{4 i}\right)_{\min }=\sum_{i=1}^{L} w_{h}^{2} \gamma_{h} S_{y h}^{2}\left(1-\rho_{c}^{2}\right)$

where, $\rho_{c}$ is combined correlation coefficient in stratified sampling across all

strata. It is calculated as $\rho_{c}^{2}=\frac{\left(\sum_{i=1}^{L} w_{h}^{2} \gamma_{h} \rho_{h} S_{y h} S_{x h}\right)^{2}}{\sum_{1=1}^{L} w_{h}^{2} \gamma_{h} S_{y h}^{2} \sum_{i=1}^{L} w_{h}^{2} \gamma_{h} S_{x h}^{2}}$.

\section{Suggested modified exponential ratio estimator}

In stratified random sampling, Kadilar and Cingi (2005) introduced a ratio estimator as

$$
\mathrm{t}_{5}=\mathrm{k} \mathrm{t}_{1} \text {, }
$$

where the constant $k$ is obtained by minimizing the MSE of $t_{5}$.

Motivated by Kadilar and Cingi (2005), we propose the following modified estimator

$$
\begin{aligned}
\mathrm{t}_{6 \mathrm{i}} & =\mathrm{k} \mathrm{t}_{4 \mathrm{i}} \\
& =\mathrm{k} \overline{\mathrm{y}}_{\text {st }} \exp \left[\frac{\overline{\mathrm{X}}_{4 \mathrm{i}}-\overline{\mathrm{x}}_{4 \mathrm{i}}}{\overline{\mathrm{X}}_{4 \mathrm{i}}+\overline{\mathrm{x}}_{4 \mathrm{i}}}\right], \quad \mathrm{i}=0,1,2,3,4 .
\end{aligned}
$$

To obtain the MSE of $t_{6 i}$ to the first degree of approximation, let us define

$$
\begin{gathered}
\mathrm{e}_{0}=\frac{\overline{\mathrm{y}}_{\mathrm{st}}-\overline{\mathrm{Y}}}{\overline{\mathrm{Y}}} \text { and } \mathrm{e}_{1 \mathrm{i}}^{*}=\frac{\mathrm{a}_{\mathrm{i}}^{*}-\mathrm{A}_{\mathrm{i}}^{*}}{\mathrm{~A}_{\mathrm{i}}^{*}} \text {. Using these notations we have } \\
\overline{\mathrm{y}}_{\mathrm{st}}=\overline{\mathrm{Y}}\left(1+\mathrm{e}_{0}\right) \text { and } \mathrm{a}_{\mathrm{i}}^{*}=\mathrm{A}_{\mathrm{i}}^{*}\left(1+\mathrm{e}_{1 \mathrm{i}}^{*}\right),
\end{gathered}
$$


where

$$
\begin{aligned}
& \mathrm{A}_{\mathrm{i}}^{*}=\sum_{\mathrm{h}=1}^{\mathrm{L}} \mathrm{w}_{\mathrm{h}} \mathrm{a}_{\mathrm{hi}} \bar{X}_{\mathrm{h}}, \quad \mathrm{a}_{\mathrm{i}}^{*}=\sum_{\mathrm{h}=1}^{\mathrm{L}} \mathrm{w}_{\mathrm{h}} \mathrm{a}_{\mathrm{hi}} \overline{\mathrm{x}}_{\mathrm{h}}, \\
& \mathrm{E}\left(\mathrm{e}_{0}\right)=\mathrm{E}\left(\mathrm{e}_{1 \mathrm{i}}^{*}\right)=0 \\
& \mathrm{E}\left(\mathrm{e}_{0}^{2}\right)=\frac{1}{\overline{\mathrm{Y}}^{2}} \sum_{\mathrm{h}=1}^{\mathrm{L}} \mathrm{w}_{\mathrm{h}}^{2} \theta_{\mathrm{h}} \mathrm{S}_{\mathrm{yh}}^{2}, \\
& \mathrm{E}\left(\mathrm{e}_{1 \mathrm{i}}^{2}\right)=\frac{1}{\mathrm{~A}_{\mathrm{i}}^{* 2}} \sum_{\mathrm{h}=1}^{\mathrm{L}} \mathrm{w}_{\mathrm{h}}^{2} \gamma_{\mathrm{h}} \mathrm{a}_{\mathrm{hi}} \mathrm{S}_{\mathrm{xh}}^{2} \\
& \mathrm{E}\left(\mathrm{e}_{0} \mathrm{e}_{1 \mathrm{i}}^{*}\right)=\frac{1}{\overline{\mathrm{Y}} \mathrm{A}_{\mathrm{i}}^{*}} \sum_{\mathrm{h}=1}^{\mathrm{L}} \mathrm{w}_{\mathrm{h}}^{2} \gamma_{\mathrm{h}} \mathrm{a}_{\mathrm{hi}_{\mathrm{i}}} \mathrm{S}_{\mathrm{yxh}} .
\end{aligned}
$$

Expressing (3.2) in terms of e's, we have

$$
\begin{aligned}
& \mathrm{t}_{6 \mathrm{i}}=\mathrm{k}_{\mathrm{i}} \overline{\mathrm{Y}}\left(1+\mathrm{e}_{0}\right) \exp \left[-\frac{\theta^{*} \mathrm{e}_{1 \mathrm{i}}^{*}}{2}\left(1+\frac{\theta^{*} \mathrm{e}_{1 \mathrm{i}}^{*}}{2}\right)^{-1}\right] \\
& \cong \mathrm{k}_{\mathrm{i}} \overline{\mathrm{Y}}\left[1+\mathrm{e}_{0}-\frac{\theta^{*} \mathrm{e}_{1 \mathrm{i}}^{*}}{2}+\frac{3 \theta^{* 2} \mathrm{e}_{1 \mathrm{i}}^{* 2}}{8}-\frac{\theta^{*} \mathrm{e}_{0} \mathrm{e}_{1 \mathrm{i}}^{*}}{2}\right] .
\end{aligned}
$$

where $\theta_{i}^{*}=\frac{A_{i}^{*}}{\bar{X}_{4 i}}$.

$\operatorname{Bias}\left(\mathrm{t}_{6 \mathrm{i}}\right)=\mathrm{E}\left(\mathrm{t}_{6 \mathrm{i}}-\overline{\mathrm{Y}}\right)$

$$
\begin{aligned}
& =\overline{\mathrm{Y}}_{\mathrm{st}}\left(\mathrm{k}_{\mathrm{i}}-1\right)+\mathrm{k}_{\mathrm{i}} \overline{\mathrm{Y}}_{\mathrm{st}}\left(\frac{3 \theta_{\mathrm{i}}^{*} \mathrm{E}\left(\mathrm{e}_{1 \mathrm{i}}^{* 2}\right)}{8}-\frac{\theta_{\mathrm{i}}^{*} \mathrm{E}\left(\mathrm{ee}_{1 \mathrm{i}}^{*}\right)}{2}\right) \\
& =\overline{\mathrm{Y}}_{\mathrm{st}}\left(\mathrm{k}_{\mathrm{i}}-1\right)+\frac{\mathrm{k}_{\mathrm{i}}}{\overline{\mathrm{X}}_{4 \mathrm{i}}} \sum_{\mathrm{h}=1}^{\mathrm{L}} \mathrm{w}_{\mathrm{h}}^{2} \gamma_{\mathrm{h}}\left[\frac{3}{8} \mathrm{R}_{4 \mathrm{i}} \mathrm{a}_{\mathrm{hi}}^{2} \mathrm{~S}_{\mathrm{xh}}^{2}-\frac{1}{2} \mathrm{a}_{\mathrm{hi}} \mathrm{S}_{\mathrm{yxh}}\right] \\
& =\overline{\mathrm{Y}}_{\mathrm{st}}\left(\mathrm{k}_{\mathrm{i}}-1\right)+\mathrm{k}_{\mathrm{i}} \operatorname{Bias}\left(\mathrm{t}_{4 \mathrm{i}}\right)
\end{aligned}
$$

From (3.3), we have

$$
\begin{gathered}
\operatorname{MSE}\left(\mathrm{t}_{6 \mathrm{i}}\right)=\left(\mathrm{k}_{\mathrm{i}}-1\right)^{2} \overline{\mathrm{Y}}^{2}+\mathrm{k}_{\mathrm{i}}^{2} \sum_{\mathrm{h}=1}^{\mathrm{L}} \mathrm{w}_{\mathrm{h}}^{2} \gamma_{\mathrm{h}}\left(\mathrm{S}_{\mathrm{yh}}^{2}-\mathrm{R}_{4 \mathrm{i}} \mathrm{a}_{\mathrm{hi}} \mathrm{S}_{\mathrm{yxh}}+\frac{\mathrm{R}_{4 \mathrm{i}}^{2}}{4} \mathrm{a}_{\mathrm{hi}}^{2} \mathrm{~S}_{\mathrm{xh}}^{2}\right) \\
+2 \mathrm{k}_{\mathrm{i}}\left(\mathrm{k}_{\mathrm{i}}-1\right) \sum_{\mathrm{h}=1}^{\mathrm{L}} \mathrm{w}_{\mathrm{h}}^{2} \gamma_{\mathrm{h}}\left(\frac{3}{8} \mathrm{R}_{4 \mathrm{i}}^{2} \mathrm{a}_{h \mathrm{i}}^{2} \mathrm{~S}_{\mathrm{xh}}^{2}-\frac{1}{2} \mathrm{R}_{4 \mathrm{i}} \mathrm{a}_{\mathrm{hi}} \mathrm{S}_{\mathrm{yxh}}\right) .
\end{gathered}
$$


MSE of the estimator $t_{6 i}$ can be rewritten as

$$
\operatorname{MSE}\left(\mathrm{t}_{6 \mathrm{i}}\right)=\left(\mathrm{k}_{\mathrm{i}}-1\right)^{2} \overline{\mathrm{Y}}^{2}+\mathrm{k}_{\mathrm{i}}^{2} \operatorname{MSE}\left(\mathrm{t}_{4 \mathrm{i}}\right)+2 \mathrm{k}_{\mathrm{i}}\left(\mathrm{k}_{\mathrm{i}}-1\right) \overline{\mathrm{Y}}_{\mathrm{st}} \operatorname{Bias}\left(\mathrm{t}_{4 \mathrm{i}}\right)
$$

To obtain the optimum value of $\mathrm{k}$, we partially differentiate the expression (3.6) with respect to $\mathrm{k}$ and put it equal to zero as follows:

$$
\begin{aligned}
& \frac{\partial}{\partial \mathrm{k}} \operatorname{MSE}\left(\mathrm{t}_{6 \mathrm{i}}\right)=0, \\
& \frac{\partial}{\partial \mathrm{k}}\left[\mathrm{k}^{2} \operatorname{MSE}\left(\mathrm{t}_{4 \mathrm{i}}\right)+\left(\mathrm{k}_{\mathrm{i}}-1\right)^{2} \overline{\mathrm{Y}}^{2}+2 \mathrm{k}_{\mathrm{i}}\left(\mathrm{k}_{\mathrm{i}}-1\right) \overline{\mathrm{Y}}_{\mathrm{st}} \operatorname{Bias}\left(\mathrm{t}_{4 \mathrm{i}}\right)\right]=0, \\
& \mathrm{k}_{\mathrm{i}}^{*}=\frac{\overline{\mathrm{Y}}^{2}+\mathrm{A}_{\mathrm{i}}}{\overline{\mathrm{Y}}^{2}+\mathrm{MSE}\left(\mathrm{t}_{4 \mathrm{i}}\right)+2 \mathrm{~A}_{\mathrm{i}}}, \quad \mathrm{i}=0,1,2,3,4 .
\end{aligned}
$$

where

$$
A_{i}=\bar{Y}_{\text {st }} \operatorname{Bias}\left(t_{4 i}\right)=\sum_{h=1}^{L} w_{h}^{2} \gamma_{h}\left(\frac{3}{8} R_{4 i}^{2} a_{h i}^{2} S_{x h}^{2}-\frac{1}{2} R_{4 i} a_{h i} S_{y x h}\right) .
$$

Note that $0<\mathrm{k}_{\mathrm{i}}^{*}<1$.

\section{Remark 2}

Shahbaz and Hanif (2009) proposed a general class of shrinkage estimator in survey sampling as

$$
\hat{\mathrm{t}}_{\mathrm{s}}=\frac{\hat{\mathrm{t}}}{1+\mathrm{T}^{-2} \operatorname{MSE}(\hat{\mathrm{t}})}
$$

where $\hat{t}$ is any available estimator of parameter $T$. The minimum MSE of $\hat{t}_{s}$ reported by Shahbaz and Hanif (2009) as

$$
\operatorname{MSE}\left(\hat{\mathrm{t}}_{\mathrm{s}}\right)=\frac{\operatorname{MSE}(\hat{\mathrm{t}})}{1+\mathrm{T}^{-2} \operatorname{MSE}(\hat{\mathrm{t}})}
$$

Following Shahbaz and Hanif (2009), the estimator $t_{6 i}$ is written as

$$
\hat{\mathrm{t}}_{6 \mathrm{iS}}=\frac{\hat{\mathrm{t}}_{4 \mathrm{i}}}{1+\overline{\mathrm{Y}}^{-2} \operatorname{MSE}\left(\hat{\mathrm{t}}_{4 \mathrm{i}}\right)}, \quad \mathrm{i}=0,1,2,3,4 \text {. }
$$

and the minimum MSE of $\hat{\mathrm{t}}_{6 \mathrm{iS}}$ is given by

$$
\operatorname{MSE}\left(\mathrm{t}_{6 i S}\right)=\frac{\operatorname{MSE}\left(\hat{\mathrm{t}}_{4 \mathrm{i}}\right)}{1+\overline{\mathrm{Y}}^{-2} \operatorname{MSE}\left(\hat{\mathrm{t}}_{4 \mathrm{i}}\right)}, \quad \mathrm{i}=0,1,2,3,4
$$




\section{Improved estimator}

We propose classes of estimators for estimating the population mean $(\bar{Y})$, in the stratified random sampling as

$$
\begin{aligned}
t_{7 i} & =\omega_{1 i} \bar{y}_{s t}+\omega_{2 i} t_{4 i}, \quad i=0,1,2,3,4 . \\
& =\omega_{1 i} \bar{y}_{s t}+\omega_{2 i} \bar{y}_{s t} \exp \left[\frac{\bar{X}_{4 i}-\bar{x}_{4 i}}{\bar{X}_{4 i}+\bar{x}_{4 i}}\right] .
\end{aligned}
$$

where $\omega_{1 \mathrm{i}}$ and $\omega_{2 \mathrm{i}}$ are real constants. There are two choices in terms of how to select $\omega_{1 \mathrm{i}}$ and $\omega_{2 \mathrm{i}}$. Some authors, such as Kadilar and Cingi (2006), use the constraint $\omega_{1 i}+\omega_{2 i}=1$, while others use an unconstrained selection of $\omega_{1 i}$ and $\omega_{2 i}$. This latter group includes Upadhyaya et al. (1985), Singh et al. (1988) and Shabbir and Gupta (2207). These authors choose $\omega_{1 i}$ and $\omega_{2 i}$ which minimize the MSE for the proposed estimator and do not insist on having $\omega_{1 \mathrm{i}}+\omega_{2 \mathrm{i}}=1$. In this paper we have made unconstrained selection of $\omega_{1 i}$ and $\omega_{2 i}$.

Expressing (4.1) in terms of e's by using the same notations as defined earlier we have

$$
\mathrm{t}_{7 \mathrm{i}}=\omega_{1 \mathrm{i}} \overline{\mathrm{Y}}\left(1+\mathrm{e}_{0}\right)+\omega_{2 \mathrm{i}} \overline{\mathrm{Y}} \exp \left[-\frac{\theta_{\mathrm{i}}^{*} \mathrm{e}_{0}}{2}\left(1+\frac{\theta_{\mathrm{i}}^{*} \mathrm{e}_{1 \mathrm{i}}}{2}\right)^{-1}\right]
$$

Expanding the right hand side of (4.2), and retaining the terms to the first order of approximation, we have

$$
\mathrm{t}_{7 \mathrm{i}} \cong \omega_{1 \mathrm{i}} \overline{\mathrm{Y}}\left(1+\mathrm{e}_{0}\right)+\omega_{2 \mathrm{i}} \overline{\mathrm{Y}}\left(1+\mathrm{e}_{0}-\frac{\theta_{\mathrm{i}}^{*} \mathrm{e}_{1 \mathrm{i}}^{*}}{2}+\frac{3 \theta_{\mathrm{i}}^{* 2} \mathrm{e}_{1 \mathrm{i}}^{* 2}}{8}-\frac{\theta_{\mathrm{i}}^{*} \mathrm{e}_{0} \mathrm{e}_{1 \mathrm{i}}^{*}}{2}\right)
$$

The bias of the estimator $t_{7 \mathrm{i}}$, to the first order of approximation, is obtained as Bias $\left(\mathrm{t}_{7 \mathrm{i}}\right)=\mathrm{E}\left(\mathrm{t}_{7 \mathrm{i}}-\overline{\mathrm{Y}}\right)$

$$
\begin{aligned}
& \cong\left(\omega_{1 i}+\omega_{2 i}-1\right) \bar{Y}_{s t}+\omega_{2 i} \sum_{h=1}^{L} w_{h}^{2} \gamma_{h}\left(\frac{3}{8} R_{4 i}^{2} a_{h i}^{2} S_{x h}^{2}-\frac{1}{2} R_{4 i} a_{h i} S_{y x h}\right) \\
& =\left(\omega_{1 i}+\omega_{2 i}-1\right) \bar{Y}_{s t}+\omega_{2 i} \bar{Y}_{s t} \operatorname{Bias}\left(t_{4 i}\right) \\
& =\left(\omega_{1 i}+\omega_{2 i}-1\right) \bar{Y}_{s t}+\omega_{2 i} A_{i}
\end{aligned}
$$

From equation (4.4), we observe that to the first order of approximation the proposed estimator is biased.

From (4.3), squaring and than retaining the terms of e's upto power two, we have

$$
\operatorname{MSE}\left(\mathrm{t}_{7 \mathrm{i}}\right)=\mathrm{E}\left(\mathrm{t}_{7 \mathrm{i}}-\overline{\mathrm{Y}}\right)^{2}
$$




$$
\begin{aligned}
= & E\left\{\omega_{1 i} \bar{Y}\left(1+e_{0}\right)+\omega_{2 i} \bar{Y}\left(1+e_{0}-\frac{\theta_{i}^{*} e_{1 i}^{*}}{2}+\frac{3 \theta_{i}^{* 2} e_{1 i}^{* 2}}{8}-\frac{\theta_{i}^{*} e_{0} e_{1 i}^{*}}{2}\right)-\bar{Y}_{s t}\right\}^{2} \\
= & E\left\{\bar{Y}\left(\omega_{1 i}+\omega_{2 i}-1\right)+\omega_{1 i} e_{0} \bar{Y}+\omega_{2 i} \bar{Y}\left(e_{0}-\frac{\theta_{i}^{*} e_{1 i}^{*}}{2}+\frac{3 \theta_{i}^{* 2} e_{1 i}^{* 2}}{8}-\frac{\theta_{i}^{*} e_{0} e_{1 i}^{*}}{2}\right)\right\}^{2} \\
\cong & \bar{Y}^{2}\left(\omega_{1 i}+\omega_{2 i}-1\right)^{2}+\omega_{1 i}^{2} \mathrm{D}+\omega_{2 i}^{2} \operatorname{MSE}\left(t_{4 i}\right) \\
& +2 \omega_{1 i} \omega_{2 i} C_{i}+2\left(\omega_{i 1}+\omega_{2 i}-1\right) \omega_{2 i} A_{i}
\end{aligned}
$$

where

$$
C_{i}=\sum_{h=1}^{L} w_{h}^{2} \gamma_{h}\left(S_{y h}^{2}-\frac{R_{4 i}}{2} a_{h i} S_{y x h}\right) \text { and } D=\sum_{h=1}^{L} w_{h}^{2} \gamma_{h} S_{y h}^{2} .
$$

In order to find optimum value of estimator $t_{7 i}$, we differentiate MSE $\left(t_{7 i}\right)$ with respect to $\omega_{1 \mathrm{i}}$ and $\omega_{2 \mathrm{i}}$. The optimum values of $\omega_{1 \mathrm{i}}$ and $\omega_{2 \mathrm{i}}$ are

$$
\omega_{1 \mathrm{i}}^{*}=\frac{\left(\overline{\mathrm{Y}}^{2}+\mathrm{A}_{\mathrm{i}}\right)\left(\overline{\mathrm{Y}}^{2}+\mathrm{C}_{\mathrm{i}}+\mathrm{A}_{\mathrm{i}}\right)-\overline{\mathrm{Y}}^{2}\left[\overline{\mathrm{Y}}^{2}+\operatorname{MSE}\left(\mathrm{t}_{4 \mathrm{i}}\right)+2 \mathrm{~A}_{\mathrm{i}}\right]}{\left(\overline{\mathrm{Y}}^{2}+\mathrm{C}_{\mathrm{i}}+\mathrm{A}_{\mathrm{i}}\right)^{2}-\left(\overline{\mathrm{Y}}^{2}+\mathrm{D}\right)\left[\overline{\mathrm{Y}}^{2}+\operatorname{MSE}\left(\mathrm{t}_{4 \mathrm{i}}\right)+2 \mathrm{~A}_{\mathrm{i}}\right]}
$$

and

$$
\omega_{2 i}^{*}=\frac{\left(\bar{Y}^{2}+A_{i}+C_{i}\right) \bar{Y}^{2}-\left(\bar{Y}^{2}+D\right)\left(\bar{Y}^{2}+A_{i}\right)}{\left(\bar{Y}^{2}+C_{i}+A_{i}\right)^{2}-\left(\bar{Y}^{2}+D\right)\left[\bar{Y}^{2}+\operatorname{MSE}\left(t_{4 i}\right)+2 A_{i}\right]} .
$$

Using these optimum values, $\omega_{1 \mathrm{i}}^{*}$ and $\omega_{2 \mathrm{i}}^{*}$, in the place of $\omega_{1 \mathrm{i}}$ and $\omega_{2 \mathrm{i}}$ in (4.5), respectively, we get the minimum MSE of the estimator $\mathrm{t}_{7 \mathrm{i}}$.

Remark 3 - Here we would like to mention that the optimum values of $\omega_{1 \mathrm{i}}$ and $\omega_{2 \mathrm{i}}$ require information about population parameters. However, in applications, to have this information is sometimes impossible. However in repeated surveys or studies based on multiphase sampling, where information is gathered on several occasions (or based on past experience) it may be possible to guess the value of these parameters quite accurately. Even though this approach may reduce the precision, it may be satisfactory provided the relative decrease in precision is marginal. (For further details refer to Sukhatme et. al. ((1984), pp. 260), Singh and Vishwakarma (2006) and Koyuncu and Kadilar (2009(b)). 


\section{Efficiency comparisons}

For the estimators, efficiency comparisons are given below.

In this section, we first compare modified estimator $t_{6 i}$ given in (3.2), with the estimator $t_{4 i}$ given in (2.4).

$$
\begin{aligned}
& \text { MSE }\left(t_{6 i}\right)<\operatorname{MSE}\left(t_{4 i}\right), \quad i=0,1,2,3,4 \text {, } \\
& \left(\mathrm{k}_{\mathrm{i}}-1\right)^{2} \overline{\mathrm{Y}}^{2}+\mathrm{k}_{\mathrm{i}}^{2} \sum_{\mathrm{h}=1}^{\mathrm{L}} \mathrm{w}_{\mathrm{h}}^{2} \gamma_{\mathrm{h}}\left(\mathrm{S}_{\mathrm{yh}}^{2}-\mathrm{R}_{4 \mathrm{i}} \mathrm{a}_{\mathrm{h}} \mathrm{S}_{\mathrm{yxh}}+\frac{\mathrm{R}_{4 \mathrm{i}}^{2}}{4} \mathrm{a}_{\mathrm{h}}^{2} \mathrm{~S}_{\mathrm{yxh}}^{2}\right) \\
& +2 \mathrm{k}_{\mathrm{i}}\left(\mathrm{k}_{\mathrm{i}}-1\right) \sum_{\mathrm{h}=1}^{1} \mathrm{w}_{\mathrm{h}}^{2} \gamma_{\mathrm{h}}\left(\frac{3 \mathrm{R}_{4 \mathrm{i}}^{2}}{8} \mathrm{a}_{\mathrm{hi}}^{2} \mathrm{~S}_{\mathrm{xh}}^{2}-\frac{1}{2} \mathrm{R}_{4 \mathrm{i}} \mathrm{a}_{\mathrm{hi}} \mathrm{S}_{\mathrm{xyh}}\right) \\
& <\sum_{\mathrm{h}=1}^{\mathrm{L}} \mathrm{w}_{\mathrm{h}}^{2} \gamma_{\mathrm{h}}\left(\mathrm{S}_{\mathrm{yh}}^{2}-\mathrm{R}_{4 \mathrm{i}} \mathrm{a}_{\mathrm{hi}} \mathrm{S}_{\mathrm{yxh}}+\frac{\mathrm{R}_{4 \mathrm{i}}^{2}}{4} \mathrm{a}_{\mathrm{hi}}^{2} \mathrm{~S}_{\mathrm{xh}}^{2}\right) \text {, } \\
& \mathrm{k}_{\mathrm{i}}>\frac{\overline{\mathrm{Y}}^{2}-\operatorname{MSE}\left(\mathrm{t}_{4 \mathrm{i}}\right)}{\overline{\mathrm{Y}}^{2}+\operatorname{MSE}\left(\mathrm{t}_{4 \mathrm{i}}\right)+2 \mathrm{~A}_{\mathrm{i}}} \text {. }
\end{aligned}
$$

As the condition (5.1) is always satisfied, we can say that the suggested estimator is more efficient than exponential ratio estimator in stratified random sampling in all conditions. Note that when $\mathrm{i}=0, \operatorname{MSE}\left(\mathrm{t}_{40}\right)=\operatorname{MSE}\left(\mathrm{t}_{3 \mathrm{st}}\right)$.

Second, we compare the improved estimator $t_{7 i}$, given in (4.1), with estimator $t_{4 i}$, given in (2.4) as follows:

$$
\begin{aligned}
& \operatorname{MSE}\left(\mathrm{t}_{7 \mathrm{i}}\right)<\operatorname{MSE}\left(\mathrm{t}_{4 \mathrm{i}}\right), \quad \mathrm{i}=0,1,2,3,4, \\
& \overline{\mathrm{Y}}^{2}\left(\omega_{1 \mathrm{i}}+\omega_{2 \mathrm{i}}-1\right)^{2}+\omega_{1 \mathrm{i}}^{2} \mathrm{D}+\omega_{2 \mathrm{i}}^{2} \operatorname{MSE}\left(\mathrm{t}_{4 \mathrm{i}}\right)+2 \omega_{1 \mathrm{i}} \omega_{2 \mathrm{i}} \mathrm{C}_{\mathrm{i}}+2\left(\omega_{1 \mathrm{i}}+\omega_{2 \mathrm{i}}-1\right) \omega_{2 \mathrm{i}} \mathrm{A}_{\mathrm{ii}_{\mathrm{i}}} \\
& <\sum_{\mathrm{h}=1}^{\mathrm{L}} \mathrm{w}_{\mathrm{h}}^{2} \gamma_{\mathrm{h}}\left(\mathrm{S}_{\mathrm{yh}}^{2}-\mathrm{R}_{4 \mathrm{i}} \mathrm{a}_{\mathrm{hi}} \mathrm{S}_{\mathrm{yxh}}+\frac{\mathrm{R}_{4 \mathrm{i}}^{2}}{4} \mathrm{a}_{\mathrm{hi}}^{2} \mathrm{~S}_{\mathrm{xh}}^{2}\right) .
\end{aligned}
$$

Let $\left(\omega_{1 \mathrm{i}}+\omega_{2 \mathrm{i}}-1\right)=\mathrm{B}_{\mathrm{i}}$. Then the expressions can be obtained on solving as

$$
\overline{\mathrm{Y}}^{2}<\frac{\Delta_{\mathrm{li}}^{*}+\Delta_{2 \mathrm{i}}^{*}}{\Delta_{3 \mathrm{i}}^{*}},
$$

where

$$
\begin{aligned}
& \Delta_{1 \mathrm{i}}^{*}=\operatorname{MSE}\left(\mathrm{t}_{4 \mathrm{i}}\right)\left(1-\omega_{2 \mathrm{i}}^{2}\right), \\
& \Delta_{2 \mathrm{i}}^{*}=2 \mathrm{~A}_{\mathrm{i}}\left[-\omega_{2 \mathrm{i}} \mathrm{B}_{\mathrm{i}}\right]-\omega_{1 \mathrm{i}}^{2} \mathrm{D}-2 \omega_{1 \mathrm{i}} \omega_{2 \mathrm{i}} \mathrm{C}_{\mathrm{i}} \\
& \Delta_{3 \mathrm{i}}^{*}=\mathrm{B}_{\mathrm{i}}{ }^{2}
\end{aligned}
$$


Next, we compare the improved estimator $t_{7 i}$ given in (4.1) with the estimator $t_{6 i}$ given in (3.2).

$$
\begin{aligned}
& \text { MSE }\left(\mathrm{t}_{7 \mathrm{i}}\right)<\operatorname{MSE}\left(\mathrm{t}_{6 \mathrm{i}}\right), \\
& \overline{\mathrm{Y}}^{2}\left(\omega_{1 \mathrm{i}}+\omega_{2 \mathrm{i}}-1\right)^{2}+\omega_{1 \mathrm{i}}^{2} \mathrm{D}+\omega_{2 \mathrm{i}}^{2} \operatorname{MSE}\left(\mathrm{t}_{4 \mathrm{i}}\right)+2 \omega_{1 \mathrm{i}} \omega_{2 \mathrm{i}} \mathrm{C}_{\mathrm{i}}+2\left(\omega_{1 \mathrm{i}}+\omega_{2 \mathrm{i}}-1\right) \omega_{2 \mathrm{i}} \mathrm{A}_{\mathrm{i}} \\
& <\overline{\mathrm{Y}}^{2}\left(\mathrm{k}_{\mathrm{i}}-1\right)^{2}+\mathrm{k}_{\mathrm{i}}^{2} \operatorname{MSE}\left(\mathrm{t}_{4 \mathrm{i}}\right)+2 \mathrm{k}_{\mathrm{i}}\left(\mathrm{k}_{\mathrm{i}}-1\right) \sum_{\mathrm{h}=1}^{1} \mathrm{w}_{\mathrm{h}}^{2} \gamma_{\mathrm{h}}\left(\frac{3 \mathrm{R}_{4 \mathrm{i}}^{2}}{8} \mathrm{a}_{\mathrm{hi}}^{2} \mathrm{~S}_{\mathrm{xh}}^{2}-\frac{1}{2} \mathrm{R}_{4 \mathrm{i}} \mathrm{a}_{\mathrm{hi}} \mathrm{S}_{\mathrm{xyh}}\right), \\
& \overline{\mathrm{Y}}^{2}<\frac{\Delta_{\mathrm{li}}+\Delta_{2 \mathrm{i}}}{\Delta_{3 \mathrm{i}}},
\end{aligned}
$$

where

$$
\begin{aligned}
& \Delta_{1 \mathrm{i}}=\operatorname{MSE}\left(\mathrm{t}_{4 \mathrm{i}}\right)\left(\mathrm{k}_{\mathrm{i}}^{2}-\omega_{2 \mathrm{i}}^{2}\right), \\
& \Delta_{2 \mathrm{i}}=2 \mathrm{~A}_{\mathrm{i}}\left[\mathrm{k}_{\mathrm{i}}\left(\mathrm{k}_{\mathrm{i}}-1\right)-\omega_{2 \mathrm{i}} \mathrm{B}\right]-\omega_{1 \mathrm{i}}^{2} \mathrm{D}-2 \omega_{1 \mathrm{i}} \omega_{2 \mathrm{i}} \mathrm{C}_{\mathrm{i}}, \\
& \Delta_{3 \mathrm{i}}=\mathrm{B}_{\mathrm{i}}{ }^{2}-\left(\mathrm{k}_{\mathrm{i}}-1\right)^{2} .
\end{aligned}
$$

Finally, we compare the improved estimator $t_{7 \mathrm{i}}$, given in (4.1), with estimator $\operatorname{MSE}\left(\mathrm{t}_{4 \mathrm{i}}\right)_{\min }$ given in (2.8).

$$
\begin{aligned}
& \operatorname{MSE}\left(t_{7 i}\right)<\operatorname{MSE}\left(t_{4 i}\right)_{\min }, \quad i=0,1,2,3,4, \\
& \bar{Y}^{2}\left(\omega_{1 i}+\omega_{2 i}-1\right)^{2}+\omega_{1 i}^{2} D+\omega_{2 i}^{2} \operatorname{MSE}\left(t_{4 i}\right)+2 \omega_{1 i} \omega_{2 i} C_{i}+2\left(\omega_{1 i}+\omega_{2 i}-1\right) \omega_{2 i} A_{i} \\
& \quad<\sum_{h=1}^{L} w_{h}^{2} \gamma_{h} S_{y h}^{2}\left(1-\rho_{c}^{2}\right) .
\end{aligned}
$$

or

$$
\overline{\mathrm{Y}}^{2}<\frac{\Delta^{*}+\Delta_{1 \mathrm{i}}^{*}+\Delta_{2 \mathrm{i}}^{*}}{\Delta_{3 \mathrm{i}}^{*}}
$$

where,

$$
\begin{aligned}
& \Delta^{*}=\operatorname{MSE}\left(\mathrm{t}_{4 \mathrm{i}}\right)_{\min } \\
& \Delta_{\mathrm{li}}^{*}=\operatorname{MSE}\left(\mathrm{t}_{4 \mathrm{i}}\right)\left(-\omega_{2 \mathrm{i}}^{2}\right), \\
& \Delta_{2 \mathrm{i}}^{*}=2 \mathrm{~A}_{\mathrm{i}}\left[-\omega_{2 \mathrm{i}} \mathrm{B}_{\mathrm{i}}\right]-\omega_{1 \mathrm{i}}^{2} \mathrm{D}-2 \omega_{1 \mathrm{i}} \omega_{2 \mathrm{i}} \mathrm{C}_{\mathrm{ii}}, \\
& \Delta_{3_{\mathrm{i}}}^{*}=\mathrm{B}_{\mathrm{i}}{ }^{2} .
\end{aligned}
$$


In all above expressions we used optimum values of $\omega_{1 \mathrm{i}}, \omega_{2 \mathrm{i}}$, and $\mathrm{k}_{\mathrm{i}}$, i.e., $\omega_{1 \mathrm{i}}=\omega_{1 \mathrm{i}}^{*}, \omega_{2 \mathrm{i}}=\omega_{2 \mathrm{i}}^{*}$, and $\mathrm{k}_{\mathrm{i}}=\mathrm{k}_{\mathrm{i}}^{*}$, respectively.

Table 2: Data Statistics

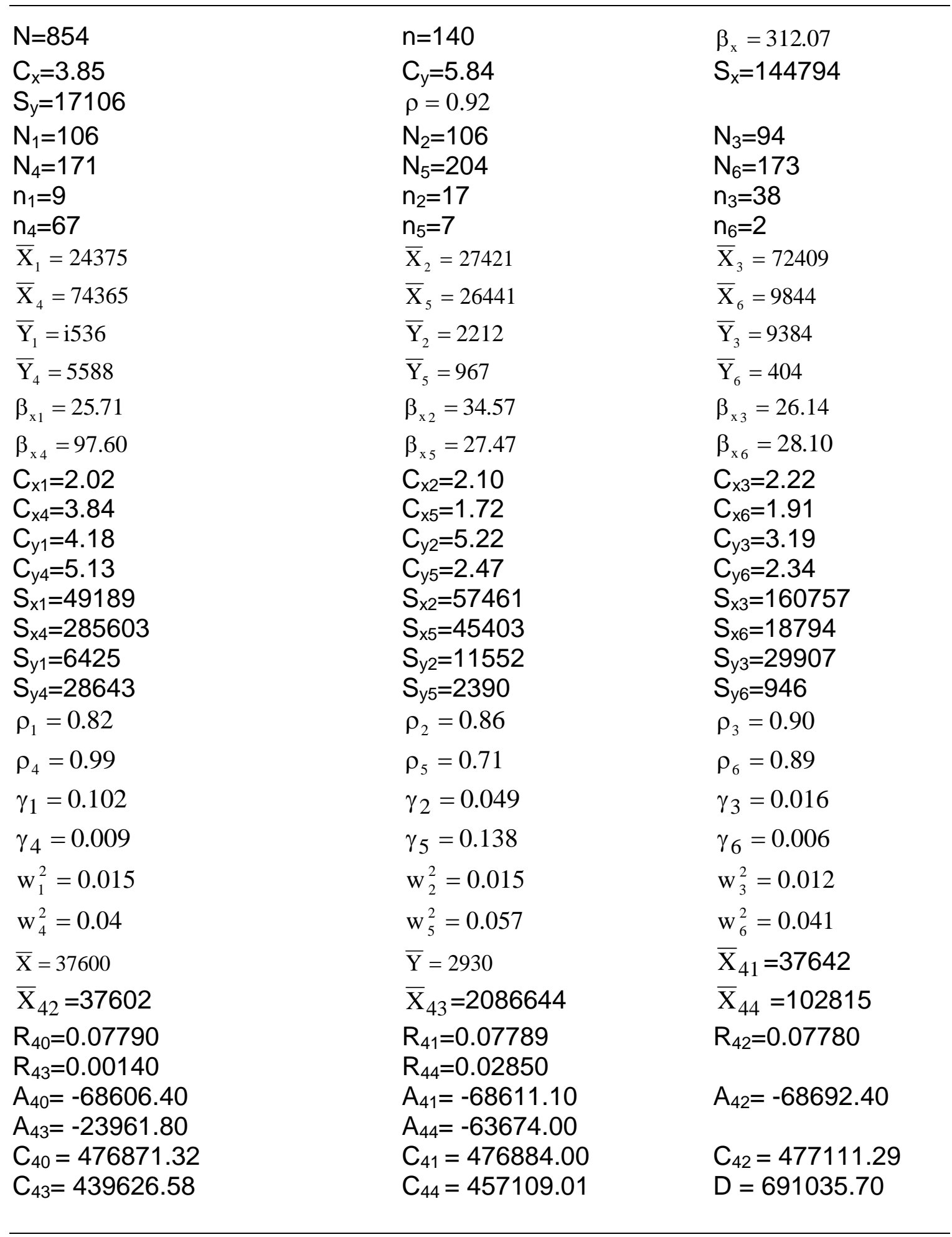




\section{Numerical study}

For empirical study, we use the data set earlier presented by Kadilar and Cingi (2003). In this data set, $Y$ is the apple production amount and $X$ is the number of apple trees in 854 villages of Turkey in 1999. The population information about this data set is given in Table 2 .

By using this data, we have calculated MSE values of suggested estimators and compared them with MSE values of Singh et al. (2008) estimators. For the different values of $a_{h}$ and $b_{h}$, we can find the MSE equations of all members of the improved estimator $\mathrm{t}_{7 \mathrm{i}}$ by simply changing $\mathrm{R}_{4 \mathrm{i}}$, respectively.

Table 3: MSE Values of Estimators

\begin{tabular}{|c|c|c|c|c|}
\hline Values of $R$ & $\begin{array}{l}\operatorname{MSE}\left(\mathbf{t}_{4 \mathbf{i}}\right) \\
(\operatorname{Bias})\end{array}$ & $\begin{array}{l}\operatorname{MSE}\left(\mathbf{t}_{6 \mathbf{6}}\right) \\
(\text { Bias) }\end{array}$ & $\begin{array}{l}\operatorname{MSE}\left(t_{7 i}\right) \\
(\text { Bias) }\end{array}$ & $\begin{array}{c}\text { Values of } \mathbf{k}_{\mathrm{i}}^{*}, \\
\omega_{1 \mathrm{i}}^{*} \text { and } \omega_{2 \mathrm{i}}^{*}\end{array}$ \\
\hline$R_{40}=0.07790$ & $\begin{array}{l}359745.60 \\
(-23.5173)\end{array}$ & $\begin{array}{l}350039.68 \\
(-\mathbf{1 1 9 . 9 8 8 7 )}\end{array}$ & $\begin{array}{l}217839.68 \\
(-74.6724)\end{array}$ & $\begin{aligned} \mathrm{k}_{0}^{*} & =0.9667 \\
\omega_{10}^{*} & =-1.1917 \\
\omega_{20}^{*} & =2.1837\end{aligned}$ \\
\hline$R_{41}=0.07789$ & $\begin{array}{l}359760.09 \\
(-23.5189)\end{array}$ & $\begin{array}{l}350053.50 \\
(\mathbf{- 1 1 9 . 9 9 3 4 )}\end{array}$ & $\begin{array}{r}217839.99 \\
(-74.6725)\end{array}$ & $\begin{aligned} \mathrm{k}_{1}^{*} & =0.9667 \\
\omega_{11}^{*} & =-1.1918 \\
\omega_{21}^{*} & =2.1839\end{aligned}$ \\
\hline $\mathrm{R}_{42}=0.07780$ & $\begin{array}{l}360008.22 \\
(-23.4940)\end{array}$ & $\begin{array}{l}350290.60 \\
(-120.0747)\end{array}$ & $\begin{array}{l}217845.36 \\
(-71.3671)\end{array}$ & $\begin{aligned} \mathrm{k}_{2}^{*} & =0.9665 \\
\omega_{12}^{*} & =-1.1942 \\
\omega_{22}^{*} & =2.1863\end{aligned}$ \\
\hline$R_{43}=0.00140$ & $\begin{array}{l}339848.98 \\
(-8.2138)\end{array}$ & $\begin{array}{l}328512.90 \\
(-112.6096)\end{array}$ & $\begin{array}{l}267874.93 \\
(-91.8238)\end{array}$ & $\begin{aligned} \mathrm{k}_{3}^{*} & =0.9641 \\
\omega_{13}^{*} & =-0.6360 \\
\omega_{23}^{*} & =1.6091\end{aligned}$ \\
\hline$R_{44}=0.0285$ & $\begin{array}{l}336684.09 \\
(-21.8266)\end{array}$ & $\begin{array}{l}328136.37 \\
(-\mathbf{1 1 2 . 4 8 0 6 )}\end{array}$ & $\begin{array}{l}208197.14 \\
(-74.6743)\end{array}$ & $\begin{array}{l}\mathrm{k}_{4}^{*}=0.9687 \\
\omega_{14}^{*}=-1.0460 \\
\omega_{24}^{*}=2.0368\end{array}$ \\
\hline
\end{tabular}

In Table 3 we have reported the bias and MSE values of the different estimators. Here we would like to mention that the MSE of the different estimators depend on choice of $a_{h}$ and $b_{h}$. The minimum MSE attained by Singh et al. (2008) estimator was 218374.8898. From Table 3 we observe that the estimator $t_{7 i}(i=0,1,2,3,4)$ 
under optimum conditions performs better than all other estimators proposed by Singh et al. (2008). We also observe from the table that the estimator $t_{73}$ is having larger MSE than the minimum MSE attained by Singh et al. (2008). So, for this data set choice of $a_{h}=\beta_{2 h}(x)$ and $b_{h}=C_{x h}$ is not a good choice. Also, for the choice $a_{h}=C_{x h}$ and $b_{h}=\beta_{2 h}(x)$ the MSEs of the estimators $t_{44}, t_{64}$ and $t_{74}$ is minimum.

\section{Conclusion}

As shown in theory, we also observe from Table 3 that suggested estimators $t_{6 i}$ (including optimal value) performs better than corresponding estimators without optimal values, $t_{4 i}$. Besides, we see that improved estimator $t_{7 \mathrm{i}}$ and its members are always more efficient than corresponding estimators (without optimal value of $\mathrm{k}_{\mathrm{i}}$ and with $\mathrm{k}_{\mathrm{i}}^{*}$ ) for this data set.

\section{Acknowledgements}

The authors are thankful to the referee for his valuable comments and suggestions regarding the improvement of the paper. The second author (Mukesh Kumar) is grateful to UGC, New Delhi, India, for providing financial assistance.

\section{References}

1. Bahl, S. and Tuteja, R.K. (1991). Ratio and product type exponential estimator. Information and Optimization Science XIII 159-163.

2. Hansen, M.H., Hurwitz, W.N. and Gurney, M. (1946). Problem and methods of the sample survey of business. Journal of American Statistical Association 41 174-189.

3. Kadilar, C. and Cingi, H. (2003). Ratio estimators in stratified random sampling, Biometrical Journal 45 (2), 218-225.

4. Kadilar, C. and Cingi, H. (2005). A new ratio estimator in stratified random sampling, Communications in Statistics: Theory and Methods 34 (3), 597602.

5. Kadilar, C., Cingi, H. (2006). Improvement in variance estimation using auxiliary information. Hacettepe Journal of Mathematical Statistics 35(1):111-115.

6. Khoshnevisan, M., Singh, R., Chauhan, P., Sawan, N., and Smarandache, F. (2007). A general family of estimators for estimating population mean using known value of some population parameter(s), Far East Journal of Theoretical Statistics 22 181-191. 
7. Koyuncu, N. and Kadilar, C. (2009(a)). Ratio and product estimators in stratified random sampling, Journal of Statistical Planning and Inference 139 (8), 2552-2558.

8. Koyuncu, N. and Kadilar, C. (2009(b)). Family of estimators of population mean using two auxiliary variables in stratified random sampling. Communications in Statistics: Theory and Methods 38 (14), 2398-2417.

9. Shabiir, J. and Gupta, S. (2007): On improvement in variance estimation using auxiliary information. Communications in Statistics: Theory and Methods 36: 2177-2185.

10. Shahbaz, M. Q. and Hanif, M. (2009): A general shrinkage estimator in survey sampling. World Applied Sciences Journal 7(5): 593-596.

11. Singh, H. P. and Vishwakarma, G. K. (2006). Combined ratio-product estimator of finite population mean in stratified sampling, Metodologia de Encuestas 8 35-44.

12. Singh, H. P. and Vishwakarma, G. K. (2008). A family of estimators of population mean using auxiliary information in stratified sampling. Communications in Statistics: Theory and Methods 37 1038-1050.

13. Singh, H. P., Upadhyaya, L. N., Namjoshi, D. (1988). Estimation of finite population variance. Current Science 57(24):1331-1334.

14. Singh, H.P. and Tailor, R. (2003). Use of known correlation coefficient in estimating the finite population mean. Statistics in Transition 6 555-560.

15. Singh, R. and Kumar, M. (2009): A note on transformations on auxiliary variable in survey sampling. Model Assisted Statistics and Applications (Accepted).

16. Singh, R., Cauhan, P., Sawan, N., and Smarandache, F. (2007). Auxiliary Information and A Priori Values in Construction of Improved Estimators. Renaissance High Press.

17. Singh, R., Kumar, M. and Smarandache, F. (2008): Almost Unbiased Estimator for Estimating Population Mean Using Known Value of Some Population Parameter(s). Pakistan Journal of Statistics and Operations Research 4(2) pp63-76.

18. Singh, R., Kumar, M., Singh, R.D., and Chaudhary, M.K. (2008): Exponential ratio type estimators in stratified random sampling, Presented in International Symposium on Optimisation and Statistics (I.S.O.S) at A.M.U., Aligarh, India, during 29-31 Dec. 2008.

19. Sisodia, B.V.S. and Dwivedi, V.K. (1981). A modified ratio estimator using coefficient of variation of auxiliary variable, Journal of Indian Society of Agricultural Statistics 33 13-18.

20. Sukhatme, P.V., Sukhatme, B.V., Sukhatme, S. and Ashok, C. (1984). Sampling Theory of Surveys with Applications. Iowa State University Press, Ames, lowa and ICAR, New Delhi. 
21. Upadhyaya, L. N., Singh, H. P., Vos, J. W. E. (1985). On the estimation of population means and ratios using supplementary information. Statistica Neerlandica 39:309-318.

22. Upadhyaya, L.N. and Singh, H.P. (1999). An estimator for population variance that utilizes the kurtosis of an auxiliary variable in sample surveys. Vikram Mathematical Journal 19 14-17. 